\title{
Effect of Oral and Maxillofacial Injuries on the Development of Post-Traumatic Stress Disorder: A Cross-Sectional Study
}

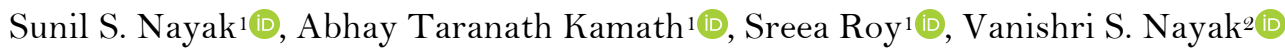

\begin{abstract}
${ }^{1}$ Department of Oral and Maxillofacial Surgery, Manipal College of Dental Sciences, Manipal Academy of Higher Education, Manipal, Karnataka, India.

2Department of Anatomy, Kasturba Medical College, Manipal Academy of Higher Education, Manipal, Karnataka, India.
\end{abstract}

Correspondence: Dr. Sunil S. Nayak, Associate Professor, Department of Oral and Maxillofacial Surgery, Manipal College of Dental Sciences, Manipal Academy of Higher Education, Manipal, Karnataka, India. E-mail: sunil.nayak@manipal.edu

Academic Editor: Alidianne Fábia Cabral Cavalcanti

Received: 04 June 2020 / Review: 28 August 2020 / Accepted: 09 October 2020

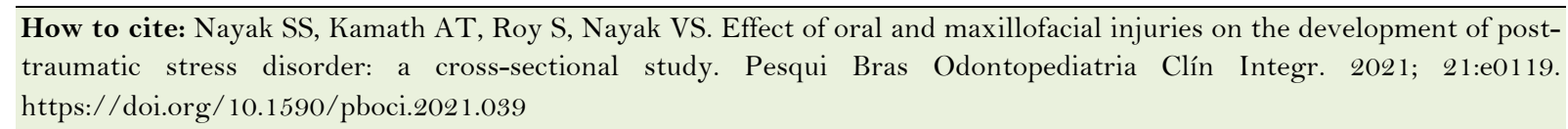

\begin{abstract}
Objective: To assess the influence of oral and maxillofacial trauma on the development of Post-Traumatic Stress Disorder (PTSD) and to determine the efficiency of the Impact of Event Scale-Revised (IES-R) as a diagnostic tool for detecting PTSD in patients with Oral and Maxillofacial injuries. Material and Methods: PTSD was assessed one month postoperatively by the diagnostic instrument, IES-R, to arrive at a provisional diagnosis. A structured clinician-administered PTSD Scale then assessed the patients for the Diagnostic and Statistical Manual of Mental Disorders-5th edition (CAPS-5) to establish a final diagnosis. The assessment of the severity of PTSD was done based on various types of oral and maxillofacial injuries. Results: The IES-R scale provisionally diagnosed 54 subjects with PTSD, out of which 42 were diagnosed to have PTSD by the CAPS-5 scale. Subjects with injuries involving the 'orbital complex,' those presenting with a perceptible scar in the maxillofacial region and with multiple avulsed/ luxated anterior teeth, showed a higher affinity to develop PTSD, and this was statistically significant. Conclusion: Higher levels of PTSD in patients with injuries to the maxillofacial region warrants correct diagnosis and detection, and hence the maxillofacial surgeon plays a vital role in this regard. The IES-R is a useful diagnostic tool to detect PTSD early.
\end{abstract}

Keywords: Maxillofacial Injuries; Stress Disorders, Post-Traumatic; Tooth Avulsion. 


\section{Introduction}

Maxillofacial deformity resulting from trauma can affect the facial skeleton, including the dentition and the covering of soft tissues. The deformities can be functional or aesthetic, resulting in severe psychological trauma with devastating consequences. Although psychological complications associated with maxillofacial trauma is a common finding, they often go undetected [1]. Studies conducted in this regard have not adequately addressed the psychological effects due to trauma to the maxillofacial region [2]. It has been shown that patients treated by maxillofacial surgery exhibited higher levels of anxiety [3]. Anxiety, depression, insomnia, and Post-Traumatic Stress Disorder (PTSD) were exhibited by many patients after encountering maxillofacial trauma [1].

Trauma-induced stress can lead to PTSD, a psychiatric condition that can severely damage the patient. In this condition, the subjects, in addition to depression, may also exhibit suicidal tendencies [4]. Some patients may meet the full criteria of PTSD symptoms, and others may have a 'partial PTSD,' wherein there is no complete fulfillment of PTSD criteria [5]. A prevalence of 10\%-30\% of chronic PTSD has been reported 12 months after the trauma [6,7]. Avoidance of thoughts/numbing, frequent re-experiencing the traumatic episode and hyperarousal are the exclusive features of PTSD [8,9].

The Diagnostic and Statistical Manual of Mental Disorders, fourth edition (DSM-IV) comprises intrusive recollections, signs of avoidance, and symptoms of hyperarousal [10]. The Impact of Event ScaleRevised (IES-R) can evaluate PTSD symptoms in various settings. Weiss and Marmar [11] modified the original impact of Event Scale (IES) questionnaire by adding 'hyperarousal,' the third important PTSD cluster, in addition to previously present 'intrusion' and 'avoidance of thoughts' diagnostic clusters. This was as per the specification of DSM-IV. The IES-R questionnaire is a good measure for assessing PTSD [12]. The use of IES-R to evaluate PTSD in maxillofacial trauma patients is rare, and its efficiency in this regard is not known. This study aimed to estimate the influence of different types of maxillofacial facial injuries on the development of PTSD in an Indian population and determine the efficiency of the IES-R as a diagnostic instrument for determining PTSD.

\section{Material and Methods}

Ethical Clearance

This study abides by the Helsinki Declaration and ethical principles regarding human experimentation. This research study project was carried out after approval by the Institutional Ethics Committee, Kasturba Hospital, Manipal, Manipal Academy of Higher Education (No: 470/ 2017).

\section{Study Design and Sample}

The registration number for this trial is CTRI/2017/11/010719, Name of the registry: Clinical Trials Registry - India (www.ctri.nic.in). One hundred forty-seven patients with all types of maxillofacial injuries, admitted in the maxillofacial surgery unit in Kasturba Hospital, Manipal, India, from August 2017 to February 2018, were considered. All participants received complete details regarding the study, and the investigators obtained informed consent from each one of them.

The study included patients with oral and maxillofacial injuries, aged between eighteen and sixty-five years, who were willing to come for a follow-up. The study excluded patients with a preexisting cognitive impairment, those on antipsychotic medication in the preceding year, and associated neurologic/ orthopedic/abdominal/chest injuries. 


\section{Data Collection}

Surgical or non-surgical management of injuries depended on various factors, including displacement of fractured segments, occlusal derangement, dental injuries, aesthetic concerns, and the age of the patient. Surgical management included open reduction and internal fixation either under general or local anesthesia. Non-surgical management included debridement, wound closure, and replacement of missing teeth. The selected patients were assessed at least one month after trauma and not later than three months post-trauma. The IES-R scale was used as the assessment tool for screening PTSD in the patients.

The IES-R has 22 items, and the patient rates each symptom as to how bothersome it had been during the preceding week. Each IES-R format has five responses (0, 1, 2, 3, 4), and it assesses 're-experiencing / intrusion,' 'avoidance / numbing,' and hyperarousal. A total IES-R cut-off score of 35 was taken as a reasonable value for the provisional PTSD diagnosis.

A final assessment by qualified experts was next done by a standard scale, the Clinician-Administered PTSD Scale for the Diagnostic and Statistical Manual of Mental Disorders-5th edition (CAPS-5), in which the patients needed to meet with the eight diagnostic criteria as per the Diagnostic and Statistical Manual of Mental Disorders, Fifth Edition (DSM-5), to be labeled positive for PTSD. Two specifications, which included delayed expression and a dissociative subtype of PTSD, were also considered to diagnose PTSD. PTSD assessment was assessed based on different types of maxillofacial injuries, which included soft tissue and dental injuries.

\section{Data Analysis}

The SPSS 22 version (IBM Corp., Armonk, NY, USA) was used in data analysis. The Pearson chisquare test was used to determine the correlation between continuous variables and the significance level was set at $5 \%$.

\section{Results}

Among 154 patients included in the study, seven patients (4.5\%) did not turn up for a follow-up. Ninety-three (63.3\%) were males and fifty-four (37\%) females. Most of the injuries were due to road traffic accidents (RTA). RTA accounted for 127 patients, followed by a history of falls accounting for 15 patients. Five patients had a history of interpersonal violence (IPV). Forty-five patients (30.6\%) had 'orbital complex' injuries associated with either a Zygomaticomaxillary complex fracture or a Lefort fracture without involving the mandible. Midface fractures not associated with an orbital component accounted for 18 subjects. Mandibular fractures accounted for 75 subjects, and panfacial fractures involving both the midface and the mandible accounted for nine patients. Ninety patients $(61.2 \%)$ with an associated soft tissue injury in the maxillofacial region. Sixty-one patients had associated avulsion/luxation of anterior teeth.

The IES-R scale had 54 patients (36.7\%) above the cut-off score of 35. The final diagnosis of PTSD, as determined by CAPS-5, accounted for 42 subjects (28.6\%). All the subjects diagnosed with PTSD showed significantly higher IES-R scores.

The Pearson Chi-square test determined the influence of various types of fracture injuries on the development of PTSD. The current study found that out of 42 patients diagnosed with PTSD, 23 had fractures involving the orbital complex (ZMC fractures and Lefort 2 and 3), and this was found to be statistically significant $(\mathrm{p}<0.01)$ (Table 1). The other fracture injuries did not show any significant results. Only two 
patients with isolated midface fractures not associated with an orbital component developed PTSD. Sixteen patients with mandibular fractures and only one patient with panfacial fractures developed PTSD.

Table 1. Association of orbital complex fractures with PTSD.

\begin{tabular}{ccccc}
\hline \multirow{2}{*}{ Orbital Complex Injuries } & \multicolumn{2}{c}{ CAPS 5 } & & \\
& Absent & Present & Total & p-value \\
& $\mathrm{N}(\%)$ & $\mathrm{N}(\%)$ & $\mathrm{N}(\%)$ & \\
\hline Absent & $83(79.0)$ & $19(45.2)$ & $102(69.4)$ & $<0.001^{*}$ \\
Present & $22(21.0)$ & $23(54.8)$ & $45(30.6)$ & \\
\multicolumn{1}{c}{ Total } & $105(100.0)$ & $42(100.0)$ & $147(100.0)$ & \\
\hline *Statistically Significant. & & & &
\end{tabular}

* Statistically Significant.

Out of the 42 patients diagnosed with PTSD, 34 had a perceptible facial scar caused due to the traumatic event. The presence of a perceptible facial scar in patients following trauma and its effect on PTSD was found to be statistically relevant by the Pearson Chi-square test $(\mathrm{p}<0.01)$ (Table 2$)$.

Table 2. Association of perceptible facial scars with PTSD.

\begin{tabular}{ccccc}
\hline \multirow{2}{*}{ Perceptible Facial Scar } & Absent & Present & Total & p-value \\
& $\mathrm{N}(\%)$ & $\mathrm{N}(\%)$ & $\mathrm{N}(\%)$ & \\
\hline Absent & $49(46.7)$ & $8(19.0)$ & $57(38.8)$ & $<0.001^{*}$ \\
Present & $56(53.3)$ & $34(81.0)$ & $90(61.2)$ & \\
\multicolumn{1}{c}{ Total } & $105(100.0)$ & $42(100.0)$ & $147(100.0)$ & \\
\hline *Statistically Significant. & & & &
\end{tabular}

Patients with avulsed/ luxated anterior teeth showed increased rates of PTSD. Out of 42 patients with PTSD, 29 had associated avulsed/ luxated anterior teeth and was statistically significant as determined by the Pearson Chi-square test $(\mathrm{p}<0.01)$ (Table 3$)$.

Table 3. Association of avulsed/luxated anterior teeth with PTSD.

\begin{tabular}{lcccc}
\hline \multirow{2}{*}{ Dental Avulsion } & \multicolumn{2}{c}{ CAPS 5 } & & \\
& Absent & Present & Total & p-value \\
& $\mathrm{N}(\%)$ & $\mathrm{N}(\%)$ & $\mathrm{N}(\%)$ & \\
\hline Absent & $73(69.5)$ & $13(31.0)$ & $86(58.5)$ & $<0.001^{*}$ \\
Present & $32(30.5)$ & $29(69.0)$ & $61(41.5)$ & \\
\multicolumn{1}{c}{ Total } & $105(100.0)$ & $42(100.0)$ & $147(100.0)$ & \\
\hline *Statistically Significant & & & &
\end{tabular}

*Statistically Significant.

\section{Discussion}

Oral and maxillofacial traumatic injuries warrant the need for psychological assessment of the affected patients requiring treatment [13]. Early identification of PTSD prevents the patient from slipping into depression and harboring any suicidal thoughts [14]. Although trauma can lead to many reactions, not all traumatic events lead to a psychological disorder like PTSD. The outcome following trauma is usually an interaction between the patient, the traumatic event, and the circumstances [15]. Some studies have reported that the role of road traffic accidents as an etiologic factor in facial bone fractures has considerably decreased, and those caused by violence and sports injuries have increased [16,17]. However, in a suburban Indian population, as was found in the current study, most of the injuries were caused by road traffic accidents. This finding is similar to a study done by Ruslin et al. [18]. 
The current study shows the high prevalence of PTSD in patients with maxillofacial injuries. In addition to assessing PTSD in maxillofacial injuries, it determines the efficiency of a diagnostic instrument, IES-R, in the diagnosis of PTSD from an oral and maxillofacial point of view by relating them to a standard structured instrument, CAPS-5. In the current study, almost $29 \%$ of patients with oral and maxillofacial injuries exhibited PTSD, and this corresponds to the study findings of Bisson et al. [19], who reported a $27 \%$ prevalence.

The use of self-report instruments has been proposed to recognize patients who are likely to develop PTSD following traumatic events [20]. The IES-R has been used with reasonable accuracy in both clinical and in large scale research studies to identify patients with PTSD [21]. Generally, there are no acceptable standard specificity and sensitivity levels. However, in the early phase following injury, to identify as many cases as possible, sensitivity may be important. These identified cases can be referred for a further diagnostic examination to determine the presence of a psychological disorder [22]. The cut-off scores were found to differ among various studies and ranged from 22 to 44 [23]. The current study considered a minimum value of sensitivity of $83 \%$. Accordingly, the corresponding cut-off scores for IES-R was 35. This cut-off score fell well within the acceptable range as determined by other studies [22-25].

Some of the factors that can lead to PTSD include loss of organs, loss of relatives, and property loss [26]. An understanding of the etiology of PTSD is essential for proper assessment and management of the condition. Patients with no family support, larger presenting scars, and even the type of trauma may lead to increased anxiety levels [27]. Ranganath et al. [1] proved in their study that one of the main risk factors in the development of PTSD is a cosmetic deformity. Moreover, disfigured/cosmetic maxillofacial injuries show higher PTSD scores [28]. Soft tissue injuries, such as lacerations and contusions, are most common in oral and maxillofacial trauma and accounted for 49.3\% and 92.1\%, respectively [29]. Mosaddad et al. [30], in their study of oral and maxillofacial trauma in motorcyclists in Iran, found that, in dental injuries, luxation and avulsion injuries accounted for $23.2 \%$ and $18.9 \%$, respectively. Luxation or avulsion of anterior teeth can be disturbing to the patient due to aesthetic concerns. In the current study, the presence of multiple avulsed anterior teeth has a significant relation to the development of PTSD. The presence of a perceptible facial scar was also significantly associated with the development of PTSD in this study. This finding corresponds to a study done by Islam et al. [31], who reported that a facial scar reinforces a negative psychological response.

The occurrence of PTSD can vary depending on the anatomic location of traumatic injuries. Unay et al. [32] found that PTSD in patients with spinal injuries is significantly higher than injuries in different anatomic locations, such as the upper limbs. From a maxillofacial perspective, in the current study, the trauma involving the 'orbital complex' plays a vital role in PTSD compared to other maxillofacial regions. In trauma involving the 'orbital complex,' certain clinical features such as circumorbital ecchymosis, circumorbital edema, subconjunctival hemorrhage, diplopia, proptosis, enophthalmos, among others, lead to increased anxiety and stress in the affected individuals and can lead to the development of PTSD. These features can affect the individual, both cosmetically and functionally.

The outcome of the current study is manifold. It is evident that, in maxillofacial trauma, the IES- R is a good diagnostic screening tools to detect PTSD. The proper knowledge about the factors playing a significant role in the development of PTSD in maxillofacial trauma, such as orbital injuries, facial lacerations, and avulsed anterior teeth, can direct maxillofacial surgeons to focus on these aspects during the surgical management of the patient. Treatment measures should be directed to minimize post-traumatic deficits by addressing both functional and esthetic deformities. Other measures to prevent suicidal intentions and to 
develop a positive attitude among the affected patients are desirable. Psychotherapy for traumatic injuries can be a useful option, along with antidepressants. Post-traumatic deficits, if inevitable, can compromise on the facial appearance of the patient and will require excellent support, psychological counseling, and a proper follow-up.

A limitation of the study is that it does not consider the mode of treatment given to the patients, as both surgical and non-surgical treatment modalities were considered. Further research is needed to determine the effect of treatment modalities in the development of PTSD.

\section{Conclusion}

The need for the correct diagnosis and early PTSD detection in maxillofacial injuries is essential, and hence, the oral and maxillofacial surgeon plays a vital role in this regard. The IES-R co-relates well with the CAPS-5 structured instrument scale. Subjects with maxillofacial injuries involving the orbital complex, those with a perceptible post-traumatic scar and avulsed anterior teeth, are more prone to develop PTSD.

\section{Authors' Contributions}

\begin{tabular}{|c|c|c|}
\hline SSN & https://orcid.org/0000-0003-4659-6500 & $\begin{array}{l}\text { Conceptualization, Methodology, Formal Analysis, Investigation, Data Curation, Writing - } \\
\text { Original Draft, Writing - Review and Editing and Supervision. }\end{array}$ \\
\hline ATK & https://orcid.org/0000-0001-64:12-3550 & Formal Analysis, Data Curation, Visualization and Supervision. \\
\hline SR & https://orcid.org/0000-0003-4390-6868 & Formal Analysis, Investigation, Data Curation, Writing - Original Draft and Visualization. \\
\hline VSN & https://orcid.org/0000-0002-9112-0491 & Writing - Original Draft, Writing - Review and Editing and Visualization. \\
\hline
\end{tabular}

\section{Financial Support}

None.

\section{Conflict of Interest}

The authors declare no conflicts of interest.

\section{Acknowledgments}

The authors would like to express their thanks to Dr. Shripathy M Bhat, Professor, Department of Psychiatry, Kasturba Medical College, Manipal, Dr. Mehul Saha, Postgraduate student, Department of Oral and Maxillofacial Surgery, Manipal College of Dental Sciences, Manipal, Dr. Kripa Gupta, Intern, Manipal College of Dental Sciences, Manipal and Dr. Anubhav Roy, Intern, Manipal College of Dental Sciences, Manipal, for all their support.

\section{Data Availability}

The data used to support the findings of this study can be made available upon request to the corresponding author.

\section{References}

[1] Ranganathan V, Panneerselvam E, Chellappazham S, Balasubramaniam S, Raja V BK. Evaluation of depression associated with post-traumatic stress disorder after maxillofacial injuries - a prospective study. J Oral Maxillofac Surg 2018; 76(6):1282.e1-1282.e9. https://doi.org/10.1016/j.joms.2018.02.011

[2] Roccia F, Dell'Acqua A, Angelini G, Berrone S. Maxillofacial trauma and psychiatric sequelae: post-traumatic stress disorder. J Craniofac Surg 2005; 16(3):355-60. https://doi.org/10.1097/01.scs.0000157028.44315.b8

[3] Hermes D, Matthes M, Saka B. Treatment anxiety in oral and maxillofacial surgery. Results of a German multicenter trial. J Craniomaxillofac Surg 2007; 35(6-7):3 16-21. https://doi.org/10.1016/j.jcms.2007.03.004

[4] Vujanovic AA, Bakhshaie J, Martin C, Reddy MK, Anestis MD. Posttraumatic stress and distress tolerance associations with suicidality in acute care psychiatric inpatients. J Nerv Ment Dis 2017; 205(7):531-41. https://doi.org/10.1097/NMD.0000000000000690 
[5] Muller M, Vandeleur C, Rodgers S, Rossler W, Castelao E, Preisig M, et al. Factors associated with comorbidity patterns in full and partial PTSD: Findings from the PsyCoLaus study. Compr Psychiatry 2014; 55(4):837-48. https://doi.org/10.1016/j.comppsych.2014.01.009.

[6] O’Donnell ML, Creamer M, Pattison P, Atkin C. Psychiatric morbidity following injury. Am J Psychiatry 2004; 161(3):507-14. https://doi.org/10.1176/appi.ajp.161.3.507

[7] Zatzick DF, Jurkovich GJ, Gentilello L, Wisner D, Rivara FP. Posttraumatic stress, problem drinking, and functional outcomes after injury. Arch Surg 2002; 137(2):200-5. https://doi.org/10.1001/archsurg.137.2.200

[8] Glynn SM, Shetty V, Elliot-Brown K, Leathers R, Belin TR, Wang J. Chronic posttraumatic stress disorder after facial injury: a 1-year prospective cohort study. J Trauma 2007; 62(2):410-18. https://doi.org/10.1097/01.ta.0000231556.05899.bo

[9] Crowley KE. Anesthetic issues and anxiety management in the female oral and maxillofacial surgery patient. Oral Maxillofac. Surg Clin North Am 2007; 19(2):141-52. https://doi.org/10.1016/j.coms.2007.01.008

[10] American Psychiatric Association. Diagnostic and statistical manual of mental disorders, 4th ed. (text revision). Washington, D.C.: American Psychiatric Association; 2000.

[11] Weiss DF, Marmer CR. The Impact of Event Scale-Revised. In: Wilson JP, Keane TM, eds. Assessing Psychological Trauma and PTSD: A Practitioners Handbook. New York: Guilford Press; 1997. p. 399-411.

[12] Weiss DS. The Impact of Event Scale-Revised. In: Wilson JP, Keane TM, eds. Assessing Psychological Trauma and PTSD: A Practitioner's Handbook. $2^{\text {nd }}$. ed. New York: Guilford Press; 2004. p. 168-189.

[13] Choudhury-Peters D, Tahim A, Davies A, Bhatti N. Model of collaborative care for post-traumatic stress disorder in patients and carers. Br J Oral Maxillofac Surg 2017; 55(4):443-4. https://doi.org/10.1016/j.bjoms.2016.1 1.002

[14] Bantjes JR, Kagee A, McGowan T, Steel H. Symptoms of posttraumatic stress, depression, and anxiety as predictors of suicidal ideation among South African university students. J Am Coll Health 2016; 64(6):429-37. https://doi.org/10.1080/07448481.2016.1178120

[15] Hull AM, Lowe T, Finlay P. The psychological impact of maxillofacial trauma: An overview of reactions to trauma. Oral Surg Oral Med Oral Pathol Oral Radiol Endod 2003; 95(5):515-20. https://doi.org/10.1067/moe.2003.161

[16] Kyrgidis A, Koloutsos G, Kommata A, Lazarides N, Antoniades K. Incidence, aetiology, treatment outcome and complications of maxillofacial fractures. A retrospective study from Northern Greece. J Craniomaxillofac Surg 2013; 41(7):637-43. https://doi.org/10.1016/j.jcms.2012.11.046

[17] Kostakis G, Stathopoulus P, Dais P, Gkinis G, Igoumenakis D, Mezitis M, et al. An epidemiologic analysis of 1142 maxillofacial fractures and concomitant injuries. Oral Surg Oral Med Oral Pathol Oral Radiol 2012; 114(5 Suppl):S69-73. https://doi.org/10.1016/j.tripleo.2011.08.029

[18] Ruslin M, Wolff J, Boffano P, Brand HS, Forouzanfar T. Dental trauma in association with maxillofacial fractures: an epidemiological study. Dent Traumatol 2015; 31(4):318-23. https://doi.org/10.1111/edt.12176

[19] Bisson JI, Shepherd JP, Dhutia M. Psychological sequelae of facial trauma. J Trauma 1997; 43(3):496-500. https://doi.org/10.1097/00005373-199709000-00018

[20] Sijbrandij M, Reitsma JB, Roberts NP, Engelhard IM, Olff M, Sonneveld LP, et al. Self-report screening instruments for post-traumatic stress disorder (PTSD) in survivors of traumatic experiences. Cochrane Database of Systematic Reviews 2013; 6. https://doi.org/10.1002/14651858.CD010575

[21] Elhai JD, Gray MJ, Kashdan TB, Franklin CL. Which instruments are most commonly used to assess traumatic event exposure and posttraumatic effects?: A survey of traumatic stress professionals. J Trauma Stress 2005; 18(5):541-5. https://doi.org/10.1002/jts.20062

[22] Morina N, Ehring T, Priebe S. Diagnostic utility of the impact of event scale-revised in two samples of survivors of war. PLoS One 2013; 8(12):e83916. https://doi.org/10.1371/journal.pone.0083916

[23] O'Donnell ML, Bryant RA, Creamer M, Carty J. Mental health following traumatic injury: toward a health system model of early psychological intervention. Clin Psychol Rev 2008; 28(3):387-406. https://doi.org/10.1016/j.cpr.2007.07.008

[24] Chen CS, Cheng CP, Yen CF, Tang TC, Yang P, Yang RC, et al. Validation of the Impact of Event Scale-Revised for adolescents experiencing the floods and mudslides. Kaohsiung J Med Sci 2011; 27(12):560-5. https://doi.org/10.1016/j.kjms.2011.06.033

[25] Bienvenu OJ, Williams JB, Yang A, Hopkins RO, Needham DM. Posttraumatic stress disorder in survivors of acute lung injury: evaluating the impact of event scale-revised. Chest 2013; 144(1):24-31. https://doi.org/10.1378/chest.12-0908

[26] Dai W, Kaminga AC, Tan H, Wang J, Lai Z, Wu X, et al. Comorbidity of post-traumatic stress disorder and anxiety in flood survivors. Prevalence and shared risk factors. Medicine (Baltimore) 2017; 96(36):e7994. https://doi.org/10.1097/MD.0000000000007994

[27] Tebble NJ, Thomas DW, Price P. Anxiety and self-consciousness in patients with minor facial lacerations. J Adv Nurs 2004; 47(4):417-26. https://doi.org/10.1111/j.1365-2648.2004.03123.x

[28] Fares Y, Fares J. Anatomical and neuropsychological effects of cluster munitions. Neurol Sci 2013; 34(12):2095-100. https://doi.org/10.1007/s10072-013-1343-7 
[29] Ferreira MC, Batista AM, Ferreira FO, Ramos-Jorge ML, Marques LS. Pattern of oral-maxillofacial trauma stemming from interpersonal physical violence and determinant factors. Dental Traumatol 2014; 30(1):15-21. https://doi.org/10.1111/edt.12047

[30] Mosaddad SA, Gheisari R, Erfani M. Oral and maxillofacial trauma in motorcyclists in an Iranian subpopulation. Dent Traumatol 2018; 34(5):347-52. https://doi.org/10.1111/edt.12428

[31] Islam S, Ahmed M, Gary M, Walton GM, Dinan TG, Hoffman GR. The association between depression and anxiety disorders following facial trauma: A comparative study. Injury 2010; 41(1):92-6. https://doi.org/10.1016/j.injury.2009.07.012

[32] Unay K, Karatoprak O, Sener N, Guven M, Bilge A. Patients with isolated musculoskeletal trauma have lower mental status scores. Clin Orthop Relat Res 2008; 466(9):2224-9. https://doi.org/10.1007/s1 1999-008-0326-0 